\title{
Análisis del cariotipo del híbrido natural Aloe x spinosissima y de sus parentales Aloe arborescens y Aloe humilis, mediante bandeo cromosómico C, CMA y DAPI
}

Karyotype analysis of natural hybrid Aloe $x$ spinosissima and its parent Aloe arborescens and Aloe humilis, by C, CMA and DAPI chromosome banding

\author{
Sánchez García Ysbelia ${ }^{1,3}$ \& Raymúndez María-B ${ }^{2}$
}

\begin{abstract}
Resumen
Se estudia el cariotipo de tres especies de Aloe con bandeo cromosómico C-Giemsa, CMA (Cromomicina A ) y DAPI (4'-6-diamino-2.fenildol), con la finalidad de realizar aportes en su cariomorfometría, ubicación, tamaño de bandas, y discutir evidencias citogenéticas sobre el origen híbrido reportado en $A$. x spinossisima. Las entidades estudiadas presentan un $2 \mathrm{n}=2 \mathrm{x}=14$ con dos pares de NORs (regiones organizadoras del nucléolo) con satélites asociados y heterocromatina constitutiva CMA + DAPI- (zonas ricas en G-C). La cantidad de heterocromatina, expresada en \% de la longitud total del cariotipo (LTC), varía entre las especies: $1,92 \%$ en $A$. arborescens, $1,69 \%$ en $A$. humilis y $1,41 \%$ en $A$. x spinossisima. Se observan bandas en los brazos largos de $\mathrm{L}_{2} \mathrm{y} \mathrm{L}_{4}$ de $A$. arborescens, en $\mathrm{L}_{3} \mathrm{y} \mathrm{L}_{4}$ de $A$. humilis y en $A$. x spinosissima en el par $\mathrm{L}_{4} \mathrm{y}_{\text {en }}$ uno de los complementos de $\mathrm{L}_{2} \mathrm{y}$ uno de $\mathrm{L}_{3}$. A . x spinossisima presenta valores de la LTC haploide y cantidad de heterocromatina constitutiva, intermedios entre las especies señaladas como progenitoras. Estos resultados y la notoria heteromorfía entre los diferentes pares cromosómicos, refuerzan la hipótesis del origen $\operatorname{de} A$. x spinosissima, como especie híbrida proveniente del cruce natural de A. arborescens $\mathrm{x}$ A. humilis.

Palabras clave: A. arborescens, A. humilis, A. spinosissima, especies simpátricas, heteromorfía cromosómica, híbrido natural.

Abstract

The karyotype of three species of Aloe was studied with C-Giemsa, CMA (Chromomycin A A $_{3}$ and DAPI (4'-6-diamino-2.fenildol) chromosome banding in order to make contributions in their cariomorfometría, location, size studied bands, and discuss cytogenetic evidence of hybrid origin reported in A. x spinossisima. The entities studied have a $2 n=2 x=14$ with two pairs of NORs (nucleolar organizer regions) with associated satellites and constitutive heterochromatin CMA + DAPI- (G-C rich regions). The amount of heterochromatin, expressed in $\%$ of the total length of the karyotype (LTC), varies between species: $1.92 \%$ by A. arborescens, $1.69 \%$ in $A$. humilis and $1.41 \%$ in A. x spinossisima. The bands are observed in the long arms of $\mathrm{L}_{2}$ and $\mathrm{L}_{4}$ of $A$. arborescens, in $\mathrm{L}_{3}$ and $\mathrm{L}_{4}$ of $A$. humilis and $A$. $\mathrm{x}$ spinosissima in the pair $\mathrm{L}_{4}$ and one of the supplements $\mathrm{L}_{2}$ and $\mathrm{L}_{3}$ one. A. $\mathrm{x}$ spinossisima presents values of LTC haploid and amount of constitutive heterochromatin intermediate between species designated as progenitor. These results and the notorious heteromorphy between different chromosome pairs, supports the hypothesis of the origin of $A$. x spinosissima, as a hybrid species from the natural crossing of $A$. arborescens x A. humilis.
\end{abstract}

Key words: A. arborescens, A. humilis, A. spinosissima, sympatric species, chromosomal heteromorphy, natural hybrid.

\footnotetext{
${ }^{1}$ Universidad Pedagógica Experimental Libertador (UPEL), Lab. Botánica Sistemática, Centro de Investigación en Ciencias Naturales (CICNAT), Inst. Pedagógico de Caracas (IPC), AP 1020, Caracas, Venezuela.

${ }^{2}$ Universidad Central de Venezuela (UCV), Lab. Biosistemática y Citogenética Vegetal, Inst. Biología Experimental (IBE), Facultad de Ciencias, AP 47114, Caracas 1041-A, Venezuela.

${ }^{3}$ Autor para la correspondencia: ysbe1576@gmail.com
} 


\section{Introducción}

El género Aloe L., circunscrito en la familia Asphodelaceae Juss. (APG IV 2016), está conformado por 11 secciones (Jacobsen 1954) y 558 especies aceptadas (The Plant List 2013), formadas por hierbas en roseta con tallos conspicuos o inconspicuos, hojas suculentas, flores tubulares, con un $\mathrm{x}=7$. Debido a que muchas de sus especies [cf. Aloe vera (L.) Burm.f., Aloe arborescens Mill., A. ferox Mill.] presentan propiedades medicinales, cosmetológicas, ornamentales y en muchos casos mágico-religiosas (Holland 1978), son explotadas ampliamente a nivel mundial y por tanto la mayoría, incluyendo las especies de esta investigación, se encuentran en el apéndice II del Comercio Internacional de Especies Amenazadas de Flora y Fauna Silvestres (CITES 2015), excepto A. x sponisissima.

Aloe x spinosissima Jahand. está conformada por plantas de hasta $1 \mathrm{~m}$ de altura, originarias de Sur África, con categoría taxonómica no resuelta (The Plant List 2013), con tallos inconspicuos, hojas que se estrechan hacia los extremos, en espiral, formando rosetas, haz cóncavo y envés convexo; láminas con dientes de hasta $8 \mathrm{~mm}$ en el margen; inflorescencias no ramificadas, flores de color naranja a rojizo (Jacobsen 1954). Su porte es semejante al de $A$. arborescens, con porte robusto como se ha reportado en otros híbridos que comparten a esta especie como parental (Lee et al. 2016). Esta especie - A. x spinosissima Jahand. - junto a $A$. arborescens Mill. y $A$. humilis (L.) Mill. son especies simpátricas en el Sur de África. Considerando su comportamiento poblacional, se reporta a Aloe x spinosissima como un híbrido natural surgido de la simpatría entre $A$. humilis $\mathrm{X}$ A. arborescens (Riley \& Majumdar 1979).

No se han publicado evidencias morfológicas o citogenéticas que refuercen la hipótesis sobre el origen híbrido de esta especie y pese a que hay publicaciones sobre el cariotipo en las tres especies (cf. Resende 1937; Snoad 1951; Sharma \& Mallick 1965; Vij et al. 1980; Brandham 1971; Imery \& Caldera 2002), no hay reportes sobre la cariomorfometría de Aloe x spinosissima, ni de bandeo cromosómico en ninguna de las tres entidades. Siendo en Aloe pocos los reportes de bandeo cromosómico de fluorescencia (Shamimul \& Khanam 2005; Sánchez 2009; Baran \& Yamamoto 2012), bandeo C (Sánchez 2009; Imery 2007) y bandeo argéntico (Matos 1998; Imery 2007; Sánchez et al. 2007; Sánchez 2009).
Los estudios citogenéticos como número cromosómico, tamaño de los cromosomas, figuras meióticas, posición del centrómero y diferentes tipos de bandeo, pueden aportar datos significativos a la hora de hacer estudios sistemáticos. Poggio et al. (1999) han señalado que los sistemáticos están familiarizados con el hecho que los cromosomas son parte de un sistema dinámico que está moldeando el proceso de la evolución. Así la citogenética ha sido empleada con fines sistemáticos y evolutivos dentro de la gran diversidad de angiospermas: en Bromeliaceae (Pires et al. 2000); en el grupo Cymbidioide de las orquídeas de Brasil (Pessoa \& Guerra 2000); Araceae (Sultana et al. 2006), en especies brasileñas de Xyris, Xyridaceae (Benko 2002); en algunas especies de Alismataceae de Brasil (Costa \& Forni-Martins 2003); en Leguminosas (Seijo \& Fernández 2003; Biondo et al. 2005; Fahmy 2006), Leucocoryne, Amaryllidaceae (Souza 2015), entre otros; así como para llevar a cabo la identificación de procesos de hibridización en Lilium (Lim et al. 2001); en algunas especies e híbridos interespecíficos de Pennisetum, Poaceae (Techio et al. 2002), entre otros.

La uniformidad mantenido en el cariotipo de Aloe en cuanto a número cromosómico, tipo de cromosomas y bimodalidad (Brandham 1971) podrían complicar la identificación de cromosomas homeólogos en especies híbridos, por tanto el uso del bandeo cromosómico podría aportar información crítica importante para su identificación.

El objetivo del presente trabajo consistió en estudiar los cromosomas de $A$. x spinosissima, $A$. arborescens y $A$. humilis a partir de bandeo cromosómico (C, CMA y DAPI), con la finalidad de realizar comparaciones en cuanto a su cariomorfometría, ubicación y tamaño de bandas C, CMA y DAPI; discutir evidencias sobre el origen híbrido de $A$. x spinossisima y sus posibles progenitores, además de aportar datos a futuros estudios biosistemáticos y de fitomejoramiento.

\section{Materiales y Métodos}

El material vegetal de Aloe examinado se obtuvo de "Mesa Gardens" (A. humilis, MG3540), "Le Jardin Nature" Francia (A. x spinosissima) y de viveros comerciales de Venezuela (A. arborescens). La identificación de cada especie fue corroborada empleando las principales descripciones y claves publicadas para el género (Jacobsen 1954; Reynolds 1966; Smith \& Newton 2001). El estudio 
se realizó empleando cinco individuos de cada una de las especies seleccionadas. Las plantas se cultivaron bajo condiciones de invernadero en el Departamento de Biología de la Universidad de Oriente, Cumaná, Venezuela y en el Laboratorio de Biosistemática y Citogenética Vegetal del IBE-UCV. El estudio cuenta con el permiso certificado de CITES número 1479.

Se colectaron ápices radicales provenientes de plantas adultas, que fueron pretratados con colchicina $(0,05 \%)$ por $2,5 \mathrm{~h}$ y posteriormente fijados en $3: 1$, etanol:ácido acético glacial por $48 \mathrm{~h}$ a $4^{\circ} \mathrm{C}$ (Sánchez et al. 2007). Las preparaciones cromosómicas se realizaron por aplastamiento previa maceración con solución enzimática (Schwarzacher et al. 1980). Se aplicó la tinción triple con los fluorocromos cromomicina $\mathrm{A}_{3}$, distamicina A y 4'-6-diamidino2-fenilindol (CMA/DA/DAPI), según Schweizer (1980), con las modificaciones necesarias. A los preparados seleccionados se les agregó una gota del antibiótico Cromomicina $\mathrm{A}_{3}$ (0,5 mg/ml. 3), se incubó en una cámara húmeda a temperatura ambiente por 90 min en la oscuridad. Las láminas fueron lavadas con abundante agua destilada hasta eliminar el cubreobjeto y el exceso de colorante. Seguidamente, se agregó una gota del antibiótico Distamicina $(0,1 \mathrm{mg} / \mathrm{ml})$ y se incubó por $60 \mathrm{~min}$. Nuevamente, se realizaron lavados con $\mathrm{H}_{2} \mathrm{Od}$ y se agregó una gota de diamidino-fenil-indol (2 $\mu \mathrm{g}$ / $\mathrm{ml}$ ) con incubación por $45 \mathrm{~min}$. El montaje de las preparaciones se realizó con un medio de glicerol y tampón McIlvaine's. Las láminas se colocaron en la estufa a $37^{\circ} \mathrm{C}$ durante 4 días. Posteriomente, se guardaron a $4^{\circ} \mathrm{C}$ hasta su estudio. En el bandeo $\mathrm{C}$ se realizó una combinación entre los protocolos de Schwarzacher et al. (1980) y Moscone et al. (1993). Los preparados se incubaron en ácido acético al $45 \%$ por min a $60^{\circ} \mathrm{C}$. La reacción se detuvo con $\mathrm{H}_{2} \mathrm{Od} \mathrm{a}$ temperatura ambiente, se sumergieron las láminas en $\mathrm{Ba}(\mathrm{OH})_{2}$ saturado por $10 \mathrm{~min}$. Los preparados se incubaron en $\mathrm{SSC} 2 \mathrm{X}$ a $60^{\circ} \mathrm{C}$ por $90 \mathrm{~min}$, se eliminó el exceso con agua destilada y se colocó una gota de DAPI $(2 \mu \mathrm{g} / \mathrm{ml})$ a los preparados de $A$. arborescens, se incubó por 60 min y se realizó el montaje en un medio de glicerol y tampón McIlvaine’s. En el caso de $A$. x spinosissima y $A$. humilis se agregó una gota de Giemsa ( $2 \%$ ) preparada con buffer fosfato $(\mathrm{pH}$ $6,8)$, se incubó por 24 h en una cámara húmeda a $37^{\circ} \mathrm{C}$. Se empleó Entellan como medio de montaje para las láminas de C-Giemsa.

Los cromosomas se observaron y fotografiaron empleando un microscopio de epifluorescencia Leica DMLB equipado con una cámara digital Leica DC 250 y el analizador de imágenes Leica IM 1000. Se efectuaron mediciones cromosómicas en quince (15) placas metafásicas de cada especie estudiada. Para la descripción y ordenamiento de los cromosomas se empleó la nomenclatura de Levan et al. (1964) y Brandham (1971). Se calcularon los índices de asimetría de Stebbins (1971), Romero Zarco (1986) y Paszko (2006).

\section{Resultados}

Los resultados obtenidos en cuanto a la fórmula cariotípica, longitud total del complemento, pares de cromosomas con NORs, cantidad de heterocromatina, máximo número de bandas por complemento haploide, índice radial, índices de asimetría y centroméricos; se detallan en las Tablas 1 y 2. Las ilustraciones referentes a los núcleos interfásicos y metafases somáticas se encuentran referidas como Figuras 1 y 2 . Los idiogramas de cada una de las especies estudiadas se presentan en

Tabla 1 - Caracteres cariotípicos de tres especies de Aloe.

Table 1 - Karyotypic characters of three species of Aloe.

\begin{tabular}{|c|c|c|c|c|c|c|c|c|c|}
\hline \multirow{3}{*}{ Especie } & \multirow{3}{*}{$\begin{array}{c}\text { Fórmula } \\
\text { cariotípica (n) }\end{array}$} & \multirow{3}{*}{$\begin{array}{c}\text { Pares } \\
\text { con } \\
\text { NORs }\end{array}$} & \multirow{3}{*}{$\begin{array}{l}\text { X LTCH } \\
(\mu \mathrm{m})(\mathrm{de})\end{array}$} & \multirow{3}{*}{$\begin{array}{c}\text { Bandeo } \\
\text { C (\%) }\end{array}$} & \multirow{3}{*}{$\begin{array}{c}\text { Cantidad } \\
\text { de } \\
\text { CMA+(\%) }\end{array}$} & \multicolumn{4}{|c|}{ Índices de asimetría } \\
\hline & & & & & & \multirow{2}{*}{ Pazsko } & \multirow{2}{*}{ Stebbins } & \multicolumn{2}{|c|}{ Romero-Zarco } \\
\hline & & & & & & & & A1 & $\mathbf{A 2}$ \\
\hline A. arborescens & $\begin{array}{c}4 \mathrm{~L}(\mathrm{st})+1 \mathrm{~S}(\mathrm{~m}) \\
+2 \mathrm{~S}(\mathrm{sm})\end{array}$ & $\mathrm{L}_{2}, \mathrm{~L}_{4}$ & $\begin{array}{l}93,70 \\
(4,39)\end{array}$ & $1,30^{*}$ & 1,92 & 15,428 & 3B & 0,635 & 0,532 \\
\hline A. x spinosissima & $\begin{array}{c}3 \mathrm{~L}(\mathrm{st})+1 \mathrm{~L}(\mathrm{t}) \\
+3 \mathrm{~S}(\mathrm{sm})\end{array}$ & $\begin{array}{l}\mathrm{L}_{2}, \mathrm{~L}_{3}, \\
\mathrm{~L}_{4}\end{array}$ & $\begin{array}{l}72,60 \\
(4,76)\end{array}$ & 0,87 & 1,41 & 18,978 & $4 \mathrm{~B}$ & 0,688 & 0,537 \\
\hline A. humilis & $\begin{array}{c}2 \mathrm{~L}(\mathrm{st})+2 \mathrm{~L}(\mathrm{t}) \\
+3 \mathrm{~S}(\mathrm{sm})\end{array}$ & $\mathrm{L}_{3}, \mathrm{~L}_{4}$ & $\begin{array}{l}66,18 \\
(2,90)\end{array}$ & 1,26 & 1,69 & 24,191 & $3 \mathrm{~B}$ & 0,698 & 0,529 \\
\hline
\end{tabular}

* = C-DAPI en A. arborescens se reveló solamente en el par $\mathrm{L}_{4} ; \mathrm{n}=$ cariotipo haploide; $\mathrm{X}=$ promedio; de = desviación estándar. 
Tabla 2 - Detalles de los valores cariotípicos de las tres especies de Aloe estudiadas.

Table 2 - Details karyotype values of the three species of Aloe studied.

\begin{tabular}{|c|c|c|c|c|c|c|c|c|c|c|c|}
\hline \multirow{3}{*}{$\begin{array}{c}\text { Par } \\
\text { cromosómico }\end{array}$} & \multicolumn{3}{|c|}{ Longitud } & \multicolumn{2}{|c|}{$\mathbf{r}(\mathbf{q} / \mathbf{p})$} & & & \multicolumn{3}{|c|}{ Longitud de la banda } & \multirow{3}{*}{$\begin{array}{l}\text { Tipo de } \\
\text { banda }\end{array}$} \\
\hline & \multicolumn{2}{|c|}{ Absoluta $(\mu \mathrm{m})$} & \multirow{2}{*}{$\begin{array}{c}\text { Relativa } \\
\text { (\% de } \\
\text { LTC) }\end{array}$} & \multirow{2}{*}{$\mathbf{X}$} & \multirow{2}{*}{ (de) } & \multirow{2}{*}{\multicolumn{2}{|c|}{ Tipo }} & \multicolumn{2}{|c|}{ Absoluta $(\mu \mathrm{m})$} & \multirow{2}{*}{$\begin{array}{c}\text { Relativa } \\
*(\% \text { de } \\
\text { LTCH) }\end{array}$} & \\
\hline & $\mathbf{X}$ & (de) & & & & & & $\mathbf{X}$ & (de) & & \\
\hline \multicolumn{12}{|c|}{ Aloe arborescens } \\
\hline $\mathrm{L}_{1}$ & 19,57 & $(1,39)$ & 20,89 & 3,09 & $(0,36)$ & st & & & & & \\
\hline $\mathrm{L}_{2}$ & 20,11 & $(1,99)$ & 21,46 & 4,33 & $(0,51)$ & st & $q$ - sat & 0,92 & $(0,18)$ & 0,95 & CMA+/DAPI- \\
\hline $\mathrm{L}_{3}$ & 19,19 & $(0,79)$ & 20,49 & 3,74 & $(0,65)$ & st & & & & & \\
\hline $\mathrm{L}_{4}$ & 17,35 & $(1,23)$ & 18,52 & 4,88 & $(0,51)$ & st & q- sat & 0,87 & $(0,15)$ & 0,95 & $\mathrm{CMA}+/ \mathrm{DAPI}-$ \\
\hline $\mathrm{S}_{1}$ & 5,91 & $(0,43)$ & 6,31 & 1,64 & $(0,10)$ & $\mathrm{m}$ & & & & & \\
\hline $\mathrm{S}_{2}$ & 5,96 & $(0,48)$ & 6,36 & 2,09 & $(0,34)$ & $\mathrm{sm}$ & & & & & \\
\hline $\mathrm{S}_{3}$ & 5,60 & $(0,62)$ & 5,98 & 2,45 & $(0,27)$ & $\mathrm{sm}$ & & & & & \\
\hline \multicolumn{12}{|l|}{ Aloe humilis } \\
\hline $\mathrm{L}_{1}$ & 14,21 & $(1,18)$ & 21,47 & 3,22 & $(0,33)$ & st & & & & & \\
\hline $\mathrm{L}_{2}$ & 13,37 & $(0,54)$ & 20,21 & 6,20 & $(0,87)$ & st & & & & & \\
\hline $\mathrm{L}_{3}$ & 13,62 & $(0,43)$ & 20,59 & 7,62 & $(0,64)$ & $\mathrm{t}$ - sat & $\mathrm{q}$ & 0,54 & $(0,18)$ & 3,98 & $\mathrm{CMA} / \mathrm{DA}+$ \\
\hline $\mathrm{L}_{4}$ & 12,57 & $(0,69)$ & 19,00 & 9,10 & $(0,77)$ & $\mathrm{t}$ - sat & $\mathrm{q}$ & 0,58 & $(0,20)$ & 4,58 & CMA/DA+ \\
\hline $\mathrm{S}_{1}$ & 4,37 & $(0,29)$ & 6,61 & 1,88 & $(0,34)$ & $\mathrm{sm}$ & & & & & \\
\hline $\mathrm{S}_{2}$ & 4,17 & $(0,27)$ & 6,30 & 2,32 & $(0,28)$ & $\mathrm{sm}$ & & & & & \\
\hline $\mathrm{S}_{3}$ & 3,82 & $(0,24)$ & 5,78 & 2,51 & $(0,43)$ & $\mathrm{sm}$ & & & & & \\
\hline
\end{tabular}

Aloe x spinosissima

\begin{tabular}{|c|c|c|c|c|c|c|c|c|c|c|c|}
\hline $\mathrm{L}_{1}$ & 15,58 & $(2,11)$ & 10,92 & 2,93 & $(0,58)$ & st & & & & & \\
\hline $\mathrm{L}_{1}$, & 14,72 & $(2,36)$ & 10,31 & 3,20 & $(0,70)$ & st & & & & & \\
\hline $\mathrm{L}_{2}$ & 13,84 & $(1,61)$ & 9,69 & 4,57 & $(0,57)$ & st & $\mathrm{q}$ - sat & 0,47 & $(0,26)$ & 3,42 & $\mathrm{CMA} / \mathrm{DA}+$ \\
\hline $\mathrm{L}_{2}$ & 14,24 & $(1,54)$ & 9,98 & 4,42 & $(0,91)$ & st & & & & & \\
\hline $\mathrm{L}_{3}$ & 14,24 & $(1,30)$ & 9,98 & 5,10 & $(1,81)$ & st & $q$ - sat & 0,52 & $(0,22)$ & 3,65 & $\mathrm{CMA} / \mathrm{DA}+$ \\
\hline $\mathrm{L}_{3}$, & 15,02 & $(2,05)$ & 10,52 & 6,25 & $(2,02)$ & st & & & & & \\
\hline $\mathrm{L}_{4}$ & 13,87 & $(2,25)$ & 9,72 & 6,72 & $(2,28)$ & st & $q$ - sat & 0,52 & $(0,26)$ & 3,78 & $\mathrm{CMA} / \mathrm{DA}+$ \\
\hline $\mathrm{L}_{4}$ & 15,34 & $(1,98)$ & 10,74 & 9,29 & $(1,72)$ & $\mathrm{t}$ & $q$ - sat & 0,61 & $(0,32)$ & 4,00 & $\mathrm{CMA} / \mathrm{DA}+$ \\
\hline $\mathrm{S}_{1}$ & 4,64 & $(0,69)$ & 3,25 & 1,96 & $(0,31)$ & $\mathrm{sm}$ & & & & & \\
\hline $\mathrm{S}_{1}$ & 4,35 & $(0,26)$ & 3,04 & 2,06 & $(0,33)$ & $\mathrm{sm}$ & & & & & \\
\hline $\mathrm{S}_{2}$ & 4,76 & $(0,40)$ & 3,34 & 2,49 & $(0,30)$ & $\mathrm{sm}$ & & & & & \\
\hline $\mathrm{S}_{2}$ & 4,01 & $(0,23)$ & 2,81 & 2,10 & $(0,25)$ & $\mathrm{sm}$ & & & & & \\
\hline $\mathrm{S}_{3}$ & 4,27 & $(0,38)$ & 2,99 & 2,49 & $(0,18)$ & $\mathrm{sm}$ & & & & & \\
\hline $\mathrm{S}_{3}$ & 4,20 & $(0,49)$ & 2,94 & 2,72 & $(0,39)$ & $\mathrm{sm}$ & & & & & \\
\hline
\end{tabular}

$\mathrm{X}=$ promedio; $\mathrm{de}=$ desviación estándar; $\mathrm{LTC}=$ longitud total del complemento (haploide en A. arborescens $\mathrm{y}$ A. humilis y diploide en $A$. $\mathrm{x}$ spinosissima); $\mathrm{r}=$ radio. 
la Figura 3 y el cariograma de $A$. spinosissima en la Figura 4. No se incluyen las imágenes de bandeo C-giemsa. Con estos datos se reporta por primera vez la cariomorfometría de $A$. X spinosissima y el bandeo cromosómico de fluorescencia CMA-DAPI y bandeo $\mathrm{C}$ en las tres especies.

Las especies estudiadas presentan un número cromosómico diploide de $2 \mathrm{n}=14$ conformado por cuatro pares cromosomas grandes $\left(\mathrm{L}_{1}-\mathrm{L}_{4}\right)$ y tres pares de cromosomas pequeños $\left(\mathrm{S}_{1}-\mathrm{S}_{3}\right)$. La fórmula del cariotipo varía entre las especies, al igual que la longitud total del complemento haploide (Tabla 1). A. humilis presenta el cariotipo de menor longitud (66,18 $\mu \mathrm{m}$; de 2,90), A. arborescens exhibe el de mayor longitud $(93,70 \mu \mathrm{m}$; de 4,39$)$ y $A$. X spinosissima presenta un tamaño intermedio de 72,60 $\mu \mathrm{m}$ (de 4,76).

Las tres entidades estudiadas presentan dos pares de NORs subterminales; uno en el par $\mathrm{L}_{4}$ y el otro varía entre las especies: en el par $\mathrm{L}_{2}$ en A. arborescens, en $\mathrm{L}_{3}$ en $A$. humilis, y en dos juegos cromosómicos en $A$. x sipnosissima (en un complemento de $\mathrm{L}_{2}$ y en uno de $\mathrm{L}_{3}$ ).
Los patrones de bandeo de fluorescencia obtenidos por combinación triple (CMA/DA/ DAPI) y doble (CMA/DAPI) son generalmente patrones reversos de DA/DAPI o DAPI/AMD. La cantidad de heterocromatina constitutiva revelada con bandas $\mathrm{CMA}+$, ricas o con moderada cantidad de G-C (expresada en porcentaje de la longitud cariotípica), además de escasa varía poco entre las especies estudiadas $(1,41-1,92)$ y se encuentra asociada a las NORs y a las bandas C (Tabs. 1; 2 y Figs. 1; 3).

En relación al índice asimetría $(\mathrm{AI})$ de Pazsko (2006), A. humilis exhibe un cariotipo marcadamente asimétrico (AI $=24,191)$, coincidente con el $\mathrm{A}_{1}$ de Romero-Zarco; mientras que $A$. arborescens presenta el cariotipo menos asimétrico $(15,428)$ y $A$. X spinosissima un valor intermedio de 18,978 (Fig. 3)

En las metafases evaluadas de A. humilis, las bandas heterocromáticas fluorescentes se muestran únicamente como CMA+/DAPI-, ubicadas en los satélites y en los brazos largos en las zonas adyacentes a la constricción secundaria
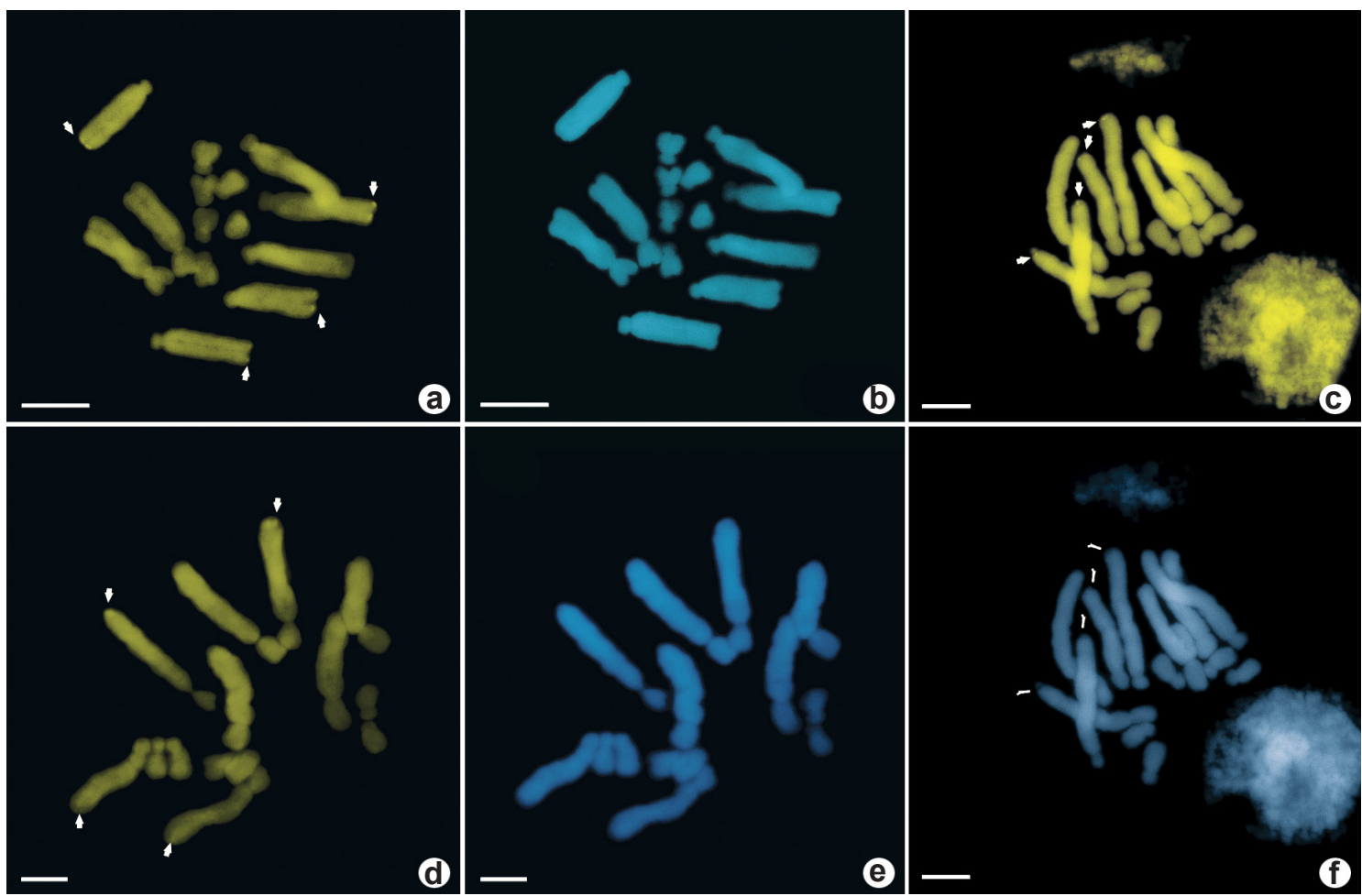

Figura 1 - Metafases somáticas, con tinción doble CMA/DAPI-a,b. A. humilis; d,e. A arborescens; c,f. A. x spinosissima. Las flechas indican bandas CMA+ (a,d,c) y las líneas blancas muestran zonas DAPI- (b,e,f). Barra $=10 \mu \mathrm{m}$.

Figure 1 - Somatic metaphases, with double staining CMA/DAPI - a,b. A. humilis; d,e. A. arborescens; c,f. A. x spinosissima. Arrows indicate bands CMA+ $(\mathrm{a}, \mathrm{d}, \mathrm{c})$ and white lines show areas DAPI- $(\mathrm{b}, \mathrm{e}, \mathrm{f}) . \mathrm{Bar}=10 \mu \mathrm{m}$. 
(Fig. 1a,b). El número de cromocentros teñidos con fluorocromos coincide perfectamente con el número de bandas observadas en los cromosomas metafásicos (Figs. 1a; 2a).

Se observan bandas C-Giemsa en las zonas subteloméricas de los pares $\mathrm{L}_{3} \mathrm{y} \mathrm{L}_{4}$, coincidente con las bandas CMA+/DAPIreveladas (Fig. 1a). Con respecto a la cantidad de heterocromatina cuantificada, ésta es de 1,26\%, siendo un porcentaje bastante cercano al 1,69\% de heterocromatina constitutiva rica en G-C revelada con bandeo de fluorescencia (Tab. 1).

A. arborescens presenta mayor cantidad de heterocromatina constitutiva, con respecto a las otras especies estudiadas $(1,92 \%)$, rica en G-C y localizada sobre los satélites de los brazos largos de los cromosomas $\mathrm{L}_{2}$ y $\mathrm{L}_{4}$, observándose en algunos casos en la zona del satélite, un gran "gap" con tinción DAPI (Fig. 1e). En algunas metafases se revela una banda DAPI+ como dos puntos en el centrómero de un cromosoma $\mathrm{S}$ y una banda CMA+ en el brazo corto de uno de los cromosomas pequeños. Las bandas $\mathrm{C}$ reveladas son ricas en G-C y pobres en A-T (Fig. 1d,e). El número de cromocentros C-DAPI determinados en los núcleos interfásicos corresponde a la cantidad de bandas observadas en las placas metafásicas.

A. x spinosissima exhibe un cariotipo de ocho cromosomas grandes (seis subtelocéntricos y dos telocéntricos) y seis cromosomas pequeños submetacéntricos. La presencia de un par telocéntrico es compartido con uno de sus posibles parentales, A. humilis, carácter poco frecuente en el género Aloe (Fig. 3). La especie presenta constricciones secundarias en uno de los complementos de $\mathrm{L}_{2} \mathrm{y} \mathrm{L}_{3}$, y en el par $\mathrm{L}_{4}$, con $1,41 \%$ de heterocromatina constitutiva rica en G-C (Tab. 1) revelada por la presencia de bandas $\mathrm{CMA}+/$ DAPI- (Fig. 1c,f), coincidentes con la presencia de bandas $\mathrm{C}$-giemsa. El número de cromocentros CMA+ es de cuatro.

\section{Discusión}

En A. humilis, Sharma \& Mallick (1965) reportan un rango del tamaño del complemento haploide entre 4,1-14,8 $\mu \mathrm{m}$, Vij et al. (1980) reportan la existencia de dos tipos citológicos de la especie, $2 \mathrm{n}=14$ y $2 \mathrm{n}=21$, con una longitud del complemento haploide entre 3,04-11,10 $\mu \mathrm{m}$ para el primer citotipo; valores cercanos a los reportados en la tabla $2(3,82-14,21 \mu \mathrm{m})$. Posteriormente, Brandham (1971) realiza el recuento cromosómico esporofítico con tinción convencional y reporta $2 \mathrm{n}=14$, sin señalar mayores cambios o diferencias en el cariotipo.

En $A$. arborescens, su cariotipo fue publicado por primera vez por Taylor (1925), quién reporta $n$ $=7$ (cuatro cromosomas grandes y tres pequeños) con constricción secundaria y satélite en un cromosoma grande. A partir de la observación de la metafase publicada por este autor y de acuerdo al tamaño del brazo corto de los cromosomas grandes, se estima que el cromosoma que exhibe la constricción secundaria es el $\mathrm{L}_{4}$. Posteriormente, Snoad (1951) confirma el nivel de ploidía y el número cromosómico $\mathrm{n}=7$.

Sharma \& Mallick (1965) trabajaron con dos variedades - A arborescens var. natalensis Berger
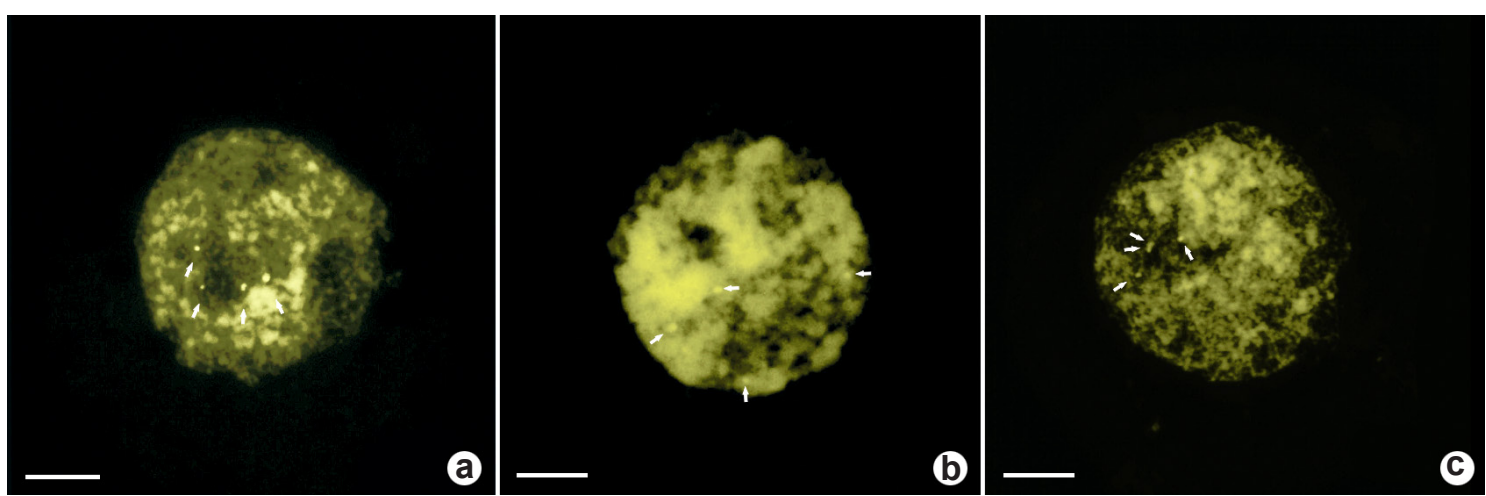

Figura 2 - Núcleos interfásicos con doble tinción CMA/DAPI - a. humilis; b. A. arborescens; c. A. x spinosissima. Los cromocentros $\mathrm{CMA}+$ están señalados con flechas blancas. Barra $=10 \mu \mathrm{m}$.

Figure 2 - Interphase nuclei with double staining CMA/DAPI-a. A. humilis; b. A. arborescens; c. A. x spinosissima. The CMA+ chromocenters are marked with white arrows. Bar $=10 \mu \mathrm{m}$. 
y A. arborescens var. milleri Berger - reconocidas como sinónimos de $A$. arborescens (The Plant List 2013). Reportan los siguientes cariotipos: $2 \mathrm{n}=14=4,5-17,4 \mu \mathrm{m}$ con variaciones de $2 \mathrm{n}$ $=13$ en $A$. arborescens var. milleri y $2 \mathrm{n}=14=$ 4,6-20 $\mu \mathrm{m}$ en A. arborescens var. natalensis. Los datos publicados por estos autores coinciden con los obtenidos en la presente investigación en el número cromosómico, presencia de constricción secundaria en el brazo largo de L y rangos de tamaños cromosómicos $(5,60-19,57 \mu \mathrm{m})$ (Tab. 2; Fig. 3).

Imery \& Caldera (2002) señalan un cariotipo conformado por un cromosoma grande sm, tres subtelocéntricos y tres pequeños submetacéntricos, cariotipo 3B y satélites en los pares $\mathrm{L}_{2} \mathrm{y} \mathrm{L}_{4}$, caracteres coincidentes con lo indicado en el presente trabajo (Tabs. 2; 3 y Fig. 3). Asimismo, es importante resaltar que las bandas C-DAPI detectadas en los brazos largos $\mathrm{L}_{4}$ coinciden con las bandas CMA+/DAPI- y con la zona 45S del ADNr (Fig. 1d,e; Adams et al. 2000; Lee et al. 2016).

\section{Heteromorfías}

Se evidencia la uniformidad mantenida en el cariotipo de Aloe, como se ha reportado en estudios previos (Brandham 1971). A. arborescens y $A$. humilis presentan cariotipos bimodales con ocho cromosomas grandes y seis pequeños. Incluso el proceso de ortoselección mencionado para el género (Brandham \& Johnson 1977) se refuerza con la presencia de bandas heterocromáticas asociadas a las zonas de organización del nucléolo en los brazos largo de los cromosomas grandes de la tres especies estudiadas (Figs. 1; 3).

Las heteromorfías observadas en esta investigación se limitan a $A$. x spinosissima, especie en la cual los cromosomas son claramente heteromorfos, como se observa en las Figuras $1 \mathrm{c}$ y $3 \mathrm{c}$. Las diferencias de mayor importancia se registran en los brazos largos de todos los cromosomas, siendo más marcadas en los pares $\mathrm{L}_{2}-\mathrm{L}_{4}$. Uno de los complementos de $\mathrm{L}_{2}$ y uno de $\mathrm{L}_{3}$ presentan bandas CMA+/DAPI- (Figs. 1c; $3 \mathrm{c}$ ) confirmadas con bandeo C-Giemsa (Fig. 2f). El par $L_{4}$, aunque presentan una notoria

\section{Aloe humilis}

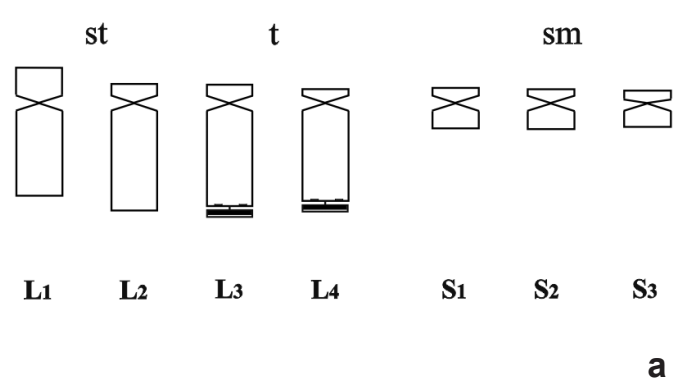

Aloe arborescens

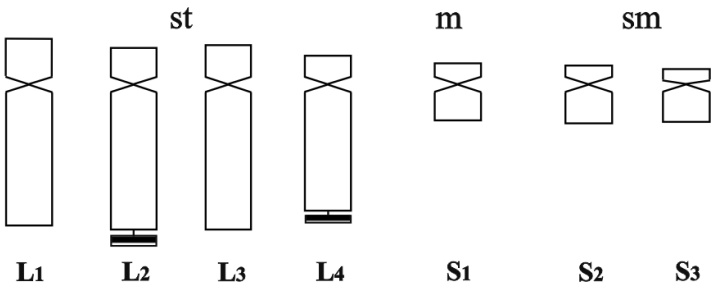

L1

\begin{abstract}
L2
\end{abstract}
$\mathbf{L 3}$

\begin{abstract}
S1
\end{abstract}
S2

Aloe spinosissima

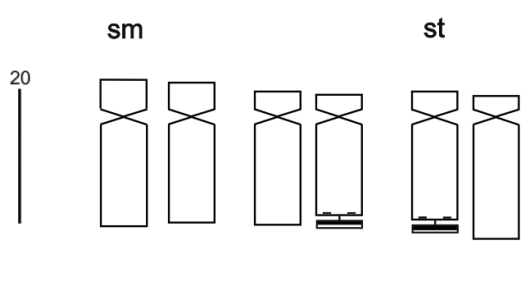

t

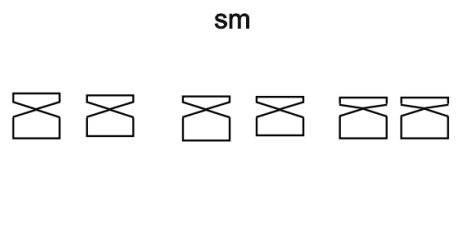

L1

L2

L3

L4

S1

S2

S3

\section{b}

Figura 3 - Figuras ideogramáticas de los cariotipos de las tres especies estudiadas. Se presentan los patrones de bandeo heterocromático con fluorocromos. Los bloques negros indican bandas CMA+/DAPI-. $(\mathrm{m}=$ metacéntrico; sm = submetacéntrico; $\mathrm{st}=$ subtelocéntrico; $\mathrm{t}=$ telocéntrico; $\mathrm{L}=$ cromosomas grandes; $\mathrm{S}=$ cromosomas pequeños). $\mathrm{Barra}=20 \mu \mathrm{m}$. Figure 3 - Ideograms of the karyotypes of the three species. Heterochromatic banding patterns with fluorocrhomes are presented. Black blocks indicate bands CMA+/DAPI-. $(\mathrm{m}=$ metacentric; $\mathrm{sm}$ = submetacentric; $\mathrm{st}=$ subtelocentric; $\mathrm{t}$ = telocentric; $\mathrm{L}=$ large chromosomes; $\mathrm{S}$ $=$ small chromosomes. Bar $=20 \mu \mathrm{m}$. 
diferencia entre el tamaño de los cromosomas, muestra bandas CMA+/DAPI- en ambos pares. Estas zonas heterocromáticas ricas en G-C y pobres en $\mathrm{A}-\mathrm{T}$, asociadas a zonas de organización del nucleolo son frecuentes en el género y se han reportado previamente en otras especies de Aloe (Shamimul \& Khanam 2005; Sánchez et al. 2009). Estas características heteromórficas entre homeólogos también se observan en los cromosomas pequeños aunque no porten bandas o constricciones secundarias que pudieran funcionar como marcadores de dichos cromosomas (Fig. 1c).

Se han reportado cambios cromosómicos estructurales dispersos en las poblaciones de una misma especie de Aloe, tales como movimientos Robertsonianos, inversiones paracéntricas y pericéntricas, translocaciones rescíprocas, deleciones, entre otros (Brandham 1969; Sapre 1978; Vij et al. 1980); observados por la formación de puentes tipo E, puentes dicéntricos, fragmentos acéntricos, bucles, entre otras aberraciones meióticas (Brandham 1969; Brandham 1977; Brandham \& Johnson 1977). Sin embargo, estas variaciones no son comunes dentro y entre especies, por acción de la selección positiva hacia la uniformidad del cariotipo - ortoselección - (Brandham \& Doherty 1998). Las variaciones mantenidas en el grupo corresponden principalmente a la presencia de heteromorfías en constricciones secundarias no compartidas entre homólogos (Sharma \& Mallick 1965; Imery \& Caldera 2002; Sánchez 2007). La presencia de cromosomas heteromorfos y la formación de figuras meióticas anormales, principalmente de puentes tipo $\mathrm{E}$, han sido señalados como delatores de procesos recientes de hibridación (Brandhan 1969, 1977; Brandham \& Johnson 1977).

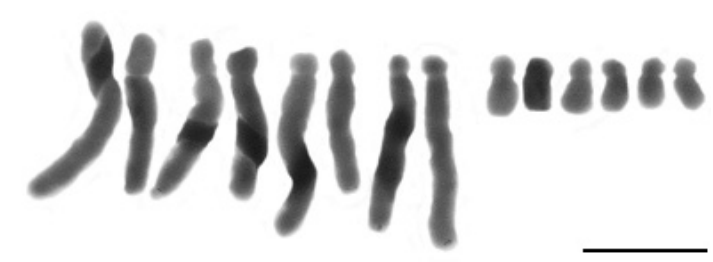

Figura 4 - Cariograma de Aloe spinosissima. La tinción se realizó con CMA. Las bandas CMA+ se observan como puntos pequeños en los subtelómeros de los cromosomas. Se observan claramente las heteromorfías entre pares homeólogos. Barra $=10 \mu \mathrm{m}$.

Figure 4-Karyotype of Aloe spinosissima with CMA staining. The CMA-positive bands are observed on chromosomes subtelomerics regions like small spots. The heteromorphy between homeologous pairs are observed. Bar $=10 \mu \mathrm{m}$.
Origen híbrido de $A$. x spinosissima

La poliploidía y la hibridación interespecífica son los fenómenos de mayor predominio en la evolución de las plantas (Friesen et al. 1997). En el género Aloe se atribuye a los procesos hibridación la principal influencia en su evolución (Reynolds 1966). A. x spinosissima es un ejemplo de ello y sus posibles progenitores son $A$. arborescens y $A$. humilis (Riley \& Majumdar 1979; Royal Botanic Gardens, Kew 2016), especies simpátricas en el sur de África, región señalada como sitio de origen de la especie estudiada (Riley \& Majumdar 1979).

Las especies señaladas como posibles parentales (Riley \& Majumdar 1979), son notoriamente diferentes en cuanto a su morfología y fueron separadas en dos grupos distantes $(A$. humilis fue circunscrita al grupo 6 y $A$. arborescens al grupo 16) debido a que $A$ humilis es de porte herbáceo con tallos inconspicuos, mientras que A. arborescens es de porte arbustivo con más de $1 \mathrm{~m}$ de altura y tallos conspicuos (Smith \& Newton 2001). Estas especies también presentan diferencias notorias a nivel citogenético (Tabs. 1; 2), los cromosomas de $A$. arborescens son de mayor tamaño $(5,60-19,57 \mu \mathrm{m})$ con respecto a los de $A$. humilis $(3,82-14,21 \mu \mathrm{m})$, haciendo una LTCH distante $(93,70$ a 63,18$)$. A arborescens muestra un cariotipo menos asimétrico $(15,428)$ que $A$. humilis $(24,191)$. Con respecto a la ubicación de las bandas, sólo coinciden en el par $\mathrm{L}_{4}$ (Fig. 4). Asimismo, estas especies se resuelven en un clado en los árboles propuestos por Treutlein et al. (2003) a partir de análisis cladísticos inferidos de secuencias del ADN cloroplástico ( $r b c \mathrm{~L}$ y matk) y la ubicación de microsatélites en el ADN nuclear, y en el grupo A propuesto por Lee et al. (2016) a partir de análisis filogenéticos basados en las secuencias nucleotídicas de psbk-psbI en 40 especies de Aloe. La coincidencia geográfica y la distancia genética son factores que influyen en el rompimiento de barreras reproductivas entre estas especies.

A. x spinosissima presenta características citogenéticas que refuerzan las hipótesis sobre su origen híbrido (Tabs. 1; 2 y Fig. 3), tales como: un cariotipo diploide heteromorfo con los pares de cromosomas grandes claramente diferentes (Figs. $3 ; 4)$, con constricciones secundarias en uno de los complementos de $\mathrm{L}_{2}$ y uno de $\mathrm{L}_{3}$, (heredados posiblemente, $\mathrm{L}_{2}$ de $A$. arborescens y $\mathrm{L}_{3}$ de $A$. humilis), valor de longitud total del complemento $(72,60 \mu \mathrm{m})$ e índices de asimetría (por ejemplo de 
Pazsko 18,978) intermedios entre sus dos parentales A. arborescens $(15,428)$ y $A$. humilis $(24,191)$.

Los resultados cariológicos y de bandeo de fluorescencia fueron corroborados con la aplicación de bandeo C. Se reveló la presencia de bandas C-Giemsa en uno de los complementos de $\mathrm{L}_{2} \mathrm{y} \mathrm{L}_{3}$, y en el par $\mathrm{L}_{4}$, detectadas como zonas ricas en $\mathrm{G}-\mathrm{C}$ con bandeo de fluorescencia (Fig. 1c,f). Las diferencias entre pares cromosómicos podrían deberse a su origen alodiploide reciente y mantenimiento de estas características por su tipo de reproducción asexual exclusiva. Lee et al. (2016) reporta resultados similares en un híbrido interespecífico entre de $A$. arborescens $\mathrm{X} A$. vera, señalado como heterocariotipo con herencia con un juego cromosómico proveniente de cada uno de sus parentales, identificados por hibridación genómica in situ (GISH) y por hibridación fluorescente in situ (FISH, ADNr 5S y 45S).

Las heteromorfías observadas a partir de la aplicación de bandeo C, CMA/DAPI y cariomorfometría; demuestran una herencia biparental del cariotipo de $A$. x spinosissima y refuerzan la importancia que han tenido los procesos de hibridación en la evolución del género.

\section{Agradecimientos}

Las autoras agradecen al Dr. José Imery del Laboratorio de Genética Vegetal de la Universidad de Oriente (UDO) por suministrar algunos de sus individuos como parte de las muestras empleadas en esta investigación.

\section{Referencias}

Adams S, Leitch L, Bennett M, Chase M \& Leitch AR (2000) Ribosomal DNA evolution and phylogeny in Aloe (Asphodelaceae). American Journal of Botany 87: 1578-1583.

APG IV - The Angiosperm Phylogeny Group (2016) An update of the Angiosperm Phylogeny Group classification for the orders and families of flowering plants. Botanical Journal of the Linnean Society 181: 1095-8339. Disponible en <http://dx.doi.org/10.1111/ boj.12385>. Acceso en 15 Agosto 2016.

Baran T \& Yamamoto M (2012) Application of EMA, fluorescent staining and FISH of rDNA in analysis of Aloe vera (1.) Burm. F. chromosome. Bulletin Faculty Agronomy Kagoshima University 62: 83-89.

Benko A \& Wanderley M (2002) Cytogenetic studies on Brazilian Xyris species (Xyridaceae). Botanical Journal of the Linnean Sciety 138: 245-252.

Biondo E, Miotto STS \& Schifino-Wittmann MT (2005) Números cromossômicos e implicações sistemáticas em espécies da subfamília Caesalpinioideae (Leguminosae) ocorrentes na região sul do Brasil. Revista Brasileira de Botânica 28: 797-808.

Brandham P (1977) The meiotic behavior of inversions in polyploid Aloineae. Chromosoma (Berl.) 62: 69-84.

Brandham PE (1969) Inversion heterozygosity and subchromatid exchange in Agave stricta. Chromosoma (Berl.) 26: 270-286.

Brandham PE (1971) The chromosome of the Liliaceae: III* New cases of interchange hybridity in the Aloineae. Kew Bulletin 28: 341-349.

Brandham P \& Doherty M (1998) Genome size variation in the Aloaceae, an angiosperm family displaying karyotypic orthoselection. Annals of Botany 82 (Suppl. A): 67-73.

Brandham P \& Johnson M (1977) Population cytology of structural and numerical chromosoma variants in the Aloïneae (Liliaceae). Plant Systematics and Evolution 128: 105-122.

Comercio Internacional de Especies Amenazadas de Flora y Fauna Silvestres (CITES) (2015) Apéndices I, II y III. Disponible en $<$ www.cites.org/esp/index. shtml $>$. Acceso en diciembre 2015.

Costa JY \& Forni-Martins ER (2003) Karyology of some Brazilian species of Alismataceae. Botanical Journal of the Linnean Sciety 143: 159-164.

Fahmy S (2006) Karyotype Analysis and Chromosome Evolution in species of Lathyrus (Fabaceae). Cytología 71: 447-455.

Friesen N, Borisjuk N, Mes T, Klaas M \& Hanelt P (1997) Allotetraploid origin of Allium altyncolicum (Alliaceae, Allium sect. Schoenoprasum) as investigated by karyological and molecular markers. PIant Systematics and Evolution 206: 317-335.

Holland PG (1978) An evolutionary biogeography of the genus Aloe. Journal Biogeography 5: 213-226.

Imery J (2007) Caracterización genética de parentales e híbridos diploides [VS] y triploides [VVS] entre Aloe vera $\mathrm{L}$. Burm. F. $[2 \mathrm{~V}, 4 \mathrm{~V}]$ y A. saponaria Haw. [2S] (Aloaceae). Tesis Doctoral. Facultad de Ciencias, Universidad Central de Venezuela, Caracas. $188 \mathrm{p}$.

Imery J \& Caldera T (2002) Estudio cromosómico comparativo de cinco especies de Aloe (Aloaceae). Acta Botánica de Venezuela 25: 47-66.

Lee YS, Park HM, Kim N, Waminal NE, Kim YJ, Lim K-B, Baek JH, Kim HH \& Yang T-J (2016) Phylogenetic relationship of 40 species of genus Aloe L. and the origin of an allodiploid species revealed by nucleotide sequence variation in chloroplast intergenic space and cytogenetic in situ hybridization. Genetic Resource and Crop Evolution 63: 235-242.

Levan A, Fredga K \& Sandberg A (1964) Nomenclatura for centromeric position on Chromosomes. Hereditas 52: 201-220.

Lim K, Wennekes J, Jong H, Jacobsen E \& Van Tuyl J (2001) Karyotype analysis of Lilium longiflorum 
and Lilium rubellum by chromosome banding and fluorescence in situ hybridization. Genome 44: 911-918.

Matos A, Molina J \& Acosta D (1998) Localización de NORs en cromosomas de Aloe vera L. (Aloaceae). Acta Botánica Venezuélica 21: 1-9.

Moscone E, Lambrou M, Hunziquer A \& Ehrendorfer F (1993) Giemsa C-banded karyotypes in Capsicum (Solanaceae). Plant Sysematics and Evolution 186: 213-229.

Paszko B (2006) A critical review and a new proposal of karyotype asymmetry indices. Plant Systematics and Evolution 258: 39-48.

Pessoa L. \& Guerra M (2000) Cytogenetics and cytotaxonomy of some Brazilian species of Cymbidioid orchids. Genetics and Molecular Biology 23: 957-978.

Pires A, Aquino J, Cortiza M, Souza J \& Silva M (2000) Chromosome numbers in Bromeliaceae. Genetics and Molecular Biology 23: 173-177.

Poggio L, Confalonieri V, Comas C, Gonzalez G \& Naranjo CA (1999) Genomic affinities of Zea luxurians, Z. diploperennis and Z. perennis: meiotic behaviour of their F1 hybrids and genomic "in situ" hybridization (GISH). Genome 42: 993-1000.

Resende F (1937) Ueber die Ubiquität der SATChoromosomen bei den Blütenpflanzen. Planta 26: 757-807.

Reynolds GW (1966) The Aloes of tropical África and Madagascar. The trustees the Aloe Book Fund, Mbabane. 351p.

Reynolds T (2004) Aloes: The genus Aloe. Medicinal and aromatic plants - industrial profiles. CRC Press, Washington D.C. 356p.

Riley HP \& Majumdar SK (1979) The Aloineae; a biosystematic survey. University Press, Kentucky. $73 \mathrm{p}$.

Romero-Zarco C (1986) A new method for estimating karyotype asymmetry. Taxon 35: 526-530.

Royal Botanic Gardens, Kew (2016) Electronic plant information centre. Disponible en $<$ http://epic.kew. org/epic/>. Acceso en 16 Agosto 2016.

Sánchez Y (2009) Contribución al conocimiento citotaxonómico en el género Aloe L. (Aloaceae) basado en patrones de bandeo cromosómico $\mathrm{C}$, AgNOR, DAPI Y CMA. Tesis Doctoral. Facultad de Ciencias, Universidad Central de Venezuela, Caracas. 243p.

Sánchez Y, Imery J \& Raymúndez MB (2007) Estandarización del protocolo AgNORs en Aloe petricola Pole Evans (Aloaceae). Revista de la Facultad de Agronomía (LUZ) 24 (Supl. 1): 1-6.
Schwarzacher T, Ambros P \& Schweizer D (1980) Application of Giemsa banding to orchid karyotype analysis. Plant Systematics and Evolution 134: 293297.

Schweizer D (1980) Simultaneous fluorescent staining of $\mathrm{R}$ bands and specific heterochromatic regions (DA DAPI) in human chromosomes. Cytogenetics and Cell Genetics 27: 190-193.

Seijo J \& Fernández A (2006) Karyotype analysis and chromosome evolution in South American species of Lathyrus (Leguminosae). American Journal of Botany 90: 980-987.

Shamimul S \& Khanam N (2005) Fluorescent karyotype analysis of four Aloe species. Bangladesh Journal of Botany 34: 17-20.

Sharma AK \& Mallick R (1965) Interrelationships and evolution of the tribe Aloineae as reflected in its cytology. Journal of Genetics 59: 110-137.

Smith GF \& Newton LE (2001) Aloaceae. In: Eggli $\mathrm{U}$ (ed.) Illustrated handbook of succulents plants: Monocotyledons. Springler, Berlin. I-XXXII illustrations [102-186]. 57p.

Snoad B (1951) Chromosome numbers of succulent plants. Heredity 5: 279-287.

Souza G, Crosa O \& Guerra M (2015) Karyological, morphological, and phylogenetic diversification in Leucocoryne Lindl (Allioideae, Amaryllidaceae). Plant Systematics and Evolution 301: 2013. Disponible en $<$ doi:10.1007/s00606-015-1216-z $>$. Acceso en Julio 2016.

Stebbins GL (1971) Chromosomal evolution in higher plants. Edward Arnold, London. 199p.

Sultana S, Bin R \& Shamimul S (2006) Karyotype analysis in three morphological forms of Lasia spinosa (L.) Thwaites (Araceae). Cytología 71: 359-364.

Taylor WR (1925) Cytological studies on Gasteria II. A comparison of the chromosomes of Gasteria, Aloe and Haworthia. American Journal of Botany 12: 219-223.

Techio V, Davide L, Pereira A \& Bearzoti E (2002) Cytotaxonomy of some species and of interespecific hybrids of Pennisetum (Poaceae, Poales). Genetics and Molecular Biologogy 25: 203-209.

The Plant List (2013) Version 1.1. Disponible en $<$ http:// www.theplantlist.org>. Acceso en 18 Agosto 2016.

Treutlein J, Smith G, Van Wyk B \& Wink M (2003) Phylogenetic relationships in Asphodelaceae (subfamily Alooideae) inferred from chloroplast DNA sequences (rbcL, matK) and from genomic fingerprinting (ISSR). Taxon 52: 193-207.

Vij SP, Sharma M \& Toor IS (1980) Cytogenetical investigation into some garden ornamentals II. The genus Aloe L. Cytologia 45: 515-532. 